\title{
Rezensionen
}

Moritz Renner, Zwingendes transnationales Recht. ZurStruktur der Wirtschaftsverfassung jenseits des Staates, Baden-Baden (NomosVerlag [Internationale Studien zur Privatrechtstheorie]) 2010, 373 Seiten, $€ 97,00$

Nicht erst mit der jüngsten Weltwirtschaftskrise ist die Notwendigkeit der Einhegung einer entgrenzten und damit politischer wie rechtlicher Kontrolle zunehmend entzogenen Ökonomie augenfällig geworden. Durch ein "opting out of the legal system" (Lisa Bernstein) können private, transnational agierende Akteure die für sie geltenden Normen weitgehend selbst bestimmen. Das Recht droht zum bloßen Instrument privatwirtschaftlicher Nutzenkalküle zu verkommen, wenn heute etwa handelsrechtliche Streitigkeiten zwischen einem deutschen und einem USamerikanischen Unternehmen von einem privaten Schiedsgericht mit Sitz in Paris nach Schweizer Recht entschieden werden. Mit dem Kontrollverlust des territorial gebundenen Nationalstaats und dem Aufstieg der globalen Wirtschaft werden die Fragen nach Möglichkeit, Gehalt und Legitimität eines Rechts der "postnationalen Konstellation“ (Habermas) immer drängender und bilden zugleich eines der fruchtbarsten Gebiete der gegenwärtigen Rechtstheorie. ${ }^{1}$

Vor diesem Hintergrund hat sich Moritz Renner mit seiner von Gralf-Peter Calliess und Gunter Teubner (als Zweitgutachter) betreuten Dissertation an der Universität Bremen keine geringere Aufgabe gestellt als die Entwicklung einer Theorie und Dogmatik zwingenden transnationalen Rechts als „Nukleus“ einer transnationalen Wirtschaftsverfassung. In einem Dreischritt führt er hierbei zunächst die wesentlichen theoretischen und dogmatischen Konzepte ein (Kapitel 1), bevor er auf der Grundlage einer Analyse der schiedsgerichtlichen Praxis (Kapitel 2) den allgemeinen und besonderen Teil einer solchen Dogmatik

1 Vgl. hierzu den Schwerpunkt Transnationales Recht, Kritische Justiz 2010, Heft 1. Dort findet sich auch ein Beitrag von Renner, Selbstgeschaffenes Recht der Wirtschaft? Öffentliche Interessen in privaten Rechtsregimes ( S. 62 ff.). des zwingenden Rechts der transnationalen Konstellation entwirft (Kapitel 3).

Es erweist sich dabei bereits die Entscheidung, an den wesentlich von Unger und Ehrlich geprägten Topos des zwingenden Rechts anzuknüpfen, als äußerst sinnvoll: Erst die Übersetzung des politischen Begriffs des Gemeinwohls in die rechtliche Kategorie des zwingenden Rechts ermöglicht nämlich eine, von der Systemtheorie als „strukturelle Kopplung" bezeichnete, wechselseitige Beeinflussung der betroffenen Systeme unter Wahrung ihrer jeweiligen Eigenrationalitäten. Zwingende Normen dienen somit dazu, politische Wertungen rechtlich zu fassen. „Ihre Aufgabe ist es, innerhalb des Privatrechts Konflikte zwischen Wirtschaft und Politik als Rechtsfragen formulierbar und entscheidbar zu machen." Als in die Sprache des Rechts übersetzte Vorrangregeln politischer Zwecksetzungen gegenüber vertraglichen Dispositionen bilden die zwingenden Normen also den Kern dessen, was von der juristischen und ökonomischen Theorie als Wirtschaftsverfassung bezeichnet wird.

Das durch das zwingende Recht vermittelte fragile Gleichgewicht zwischen ökonomischen Partikular- und politischen Gemeinwohlinteressen hängt aber wesentlich von der Normenhierarchie des territorialen Nationalstaats $a b$ und wird in grenzüberschreitenden Sachverhalten prekär: So führte bereits die Begründung des modernen Internationalen Privatrechts (IPR) durch Savignys Forderung der „freundliche[n] Zulassung [...] fremder Gesetze “2 durch den inländischen Richter zu einer Verschiebung zugunsten der Partei- und Privatautonomie. Mit den Instituten der Eingriffsnorm (z.B. im Kulturgüterschutz) und der Berücksichtigung grundlegender inländischer Wertvorstellungen (ordre public) wurden der Parteiautonomie im IPR nur sehr weite und darüber hinaus kaum konturierte Grenzen gesetzt. Selbst diese Residualkontrolle der nationalen Gesetzgeber und Gerichte aber kann durch eine strategische Wahl des Gerichtsstands (forum shopping) durch die Vertragsparteien weitgehend umgangen

2 Savigny, System des heutigen Römischen Rechts, Achter Band, 1849, S. 28. 
werden. Die Tendenz zu einer Marginalisierung staatlichen Rechts und einer damit verbundenen Aufwertung der Autonomie privater Akteure gewinnt jedoch eine neue Dimension, wenn grenzüberschreitende Streitigkeiten nicht mehr vor staatlichen Gerichten ausgetragen werden, sondern die Parteien vertraglich eine für sie oft in vielerlei Hinsicht (Vertraulichkeit, Neutralität, Kosten) vorteilhafte außergerichtliche Streitbeilegung durch Schiedsgerichte wählen. Die Entscheidungen dieser autonomen Schiedsgerichte sind dabei nicht nur für die Parteien bindend, sondern aufgrund völkerrechtlicher Verträge auch nahezu weltweit vollstreckbar. Den nationalen Gerichten verbleiben in diesem Fall als Einwirkungsmöglichkeiten lediglich noch die Aufhebung eines Schiedsspruchs im Sitzstaat des Schiedsgerichts sowie die Verweigerung der Anerkennung durch Gerichte des Vollstreckungsstaats.

Ob mit dem Aufstieg der privaten Schiedsgerichtsbarkeit, wie vielfach befürchtet, auch eine „Selbstaufgabe des Rechts als einer am gesamtgesellschaftlichen Interesse ausgerichteten Ordnung" verbunden ist, ob also zwingende Normen in den Schiedsverfahren tatsächlich keine oder kaum mehr Beachtung finden, untersucht Renner im zweiten Kapitel seiner Arbeit auf der empirischen Grundlage der Spruchpraxis dreier unterschiedlicher Regimes der Wirtschaftsschiedsgerichtsbarkeit. In der ersten Fallstudie analysiert der Autor hierfür über 200 Entscheidungen der Schiedsgerichte der Internationalen Handelskammer (ICC) in Paris. Wenngleich hier eine gegenüber dem klassischen IPR ,deutliche Ausweitung des Grundsatzes der Parteiautonomie“ zu konstatieren sei, wendeten die Schiedsrichter - häufig kollisionsrechtlich inkonsistent - auch zwingende Normen nationaler Provenienz an. Immer häufiger aber ergänzten sie diese um einen ordre public transnational, ein Korpus zwingender Rechtsregeln, die sich keiner nationalen Rechtsordnung zuordnen ließen, sondern genuin transnationaler Natur seien. In seiner negativen Funktion diene dieser dazu, die Anwendbarkeit bestimmter zwingender Normen des Schuldstatuts auszuschließen. Doch auch der positive ordre public transnational gewinne in der ICC-Praxis, etwa in Form eines allgemeinen Bestechungsverbots, zunehmend an Kontur. Eine weitaus geringere Rolle scheinen zwingende Normen dagegen für die Entscheidungen der Investitionsschiedsgerichtsbarkeit (ICSID), Gegenstand der zweiten Fallstudie, zu spielen. Dies ist wohl in erster Linie der weitgehenden Überlagerung nationalen Privatrechts durch völkerrechtliche Normen geschuldet, welche wiederum eine Reaktion auf die Asymmetrie der in Investitionsfällen beteiligten Vertragsparteien darstellt: Der Investor wäre bei strikter Anwendung nationalen Privatrechts des Anlagestaats potentiell jederzeit durch eine Enteignung bedroht. Doch auch in der ICSID-Praxis, so zeigten etwa Entscheidungen im Zusammenhang mit der Argentinienkrise, finde zumindest im Ausnahmefall eine Berücksichtigung zwingender Normen in Form des völkergewohnheitsrechtlichen Staatsnotstands statt. Weiter vorangeschritten, dies zeigt die dritte Fallstudie, ist die Herausbildung eines transnationalen zwingenden Rechts dagegen im Bereich der Domain-Name-Schiedsgerichtsbarkeit (UDRP). Ein eigenständiges Regelwerk sowie eine fortgeschrittene Veröffentlichungspraxis tragen hier zu einer Präjudizienbildung und -bindung der Schiedsgerichte bei. Die häufige Konstellation des Streits zwischen Markenrechtsinhabern und Betreibern sog. „gripesites" (z.B. „walmartsucks.com") wird hier, so die Analyse von 185 Schiedssprüchen durch Renner, vermehrt unter Rekurs auf einen allgemeinen Rechtsgrundsatz der „free speech" mit eigenständigen hochdifferenzierten Tatbestandsvoraussetzungen gelöst.

Im dritten Kapitel nun destilliert Renner aus der von ihm untersuchten Praxis die allgemeinen und regimespezifischen Grundsätze einer transnationalen Wirtschaftsverfassung. Es zeigt sich dabei, dass die Anwendung zwingenden Rechts in der transnationalen Konstellation durch eine zweigliedrig normenhierarchische Struktur gekennzeichnet ist: Ein Korpus von Sachnormen (so der ordre public transnational und die "free speech"Grundsätze), welche als internationales Einheitsrecht qualifiziert werden können, genießt Anwendungsvorrang gegenüber einer zweiten Ebene des transnationalen Rechts, welche kollisionsrechtlich ausgestaltet ist: Sekundärnormen verweisen hier auf nationale und völkerrechtliche Quellen. Der „Tod des Gesetzgebers" in der stark richterrechtlich geprägten transnationalen Konstellation macht aber auch eine methodische Neuausrichtung notwendig. Anstelle der Gesetzesbindung tritt hier die Selbstbindung von Spruchkörpern über die Argumentation mit Präjudizien. Doch eröffnet die Befassung mit der Schiedspraxis auch für die theoretische Fassung der Begriffe des transnationalen Rechts und der Wirtschaftsverfassung bedeutende Perspektiven: Schon die „scholastischen Streitigkeiten" zwischen Rechtsetatisten und Rechtspluralisten, etwa um die Frage des Rechtscharakters der lex mercatoria, erübrigten sich durch einen „Blick auf die tatsächli- 
chen Praktiken des internationalen Wirtschaftsverkehrs": Nüchtern bemerkt Renner in Anlehnung an Holmes: „The law is what the arbitrators say it is." Nur wer bereit ist, sich von alten Denkgewohnheiten, so etwa der klassischen Rechtsquellenlehre und den Dualismen von nationalem und internationalem, öffentlichem und Privatrecht, zu lösen, mag die Eigenheiten der transnationalen Konstellation erfassen: Bei dem transnationalen Recht, so nach Renner eine Konsequenz der Überwindung dieser Verhexungen des (juristischen) Verstandes, handele es sich um einen „Zusammenhang innerhalb des globalen Rechtsdiskurses, der sich von den Strukturen des nationalen Rechts und des Völkerrechts gelöst hat, aber kein eigenständiges Tertium bildet, sondern vielmehr staatliche, überstaatliche und nichtstaatliche Normenordnungen kollisionsrechtlich miteinander verschleift." Was bedeutet dies nun aber für eine transnationale Wirtschaftsverfassung? Nach Teubner scheint im autonomen Prozess vertragsbasierter Normgenese für Gesetzesrecht und, allgemeiner noch, für eine Kopplung des Rechts an die Politik „weder Bedarf zu bestehen, noch Raum zu sein." Stattdessen deute sich im transnationalen Recht "die Verwirklichung des 'liberalen Mythos' eines Vertragsrechts avant la politique" an. Allerdings, so Renner, berge Teubners politikskeptischer Ansatz "globaler Zivilverfassungen“ die Gefahr, „dass der Verrechtlichung unterschiedlicher Sozialbereiche eine apriorische Legitimation zugesprochen wird, während tatsächlich Entpolitisierungs- und Vermachtungsprozesse aus dem Blick geraten“. Diese Gefahr werde jedoch dadurch gebannt, dass eine strukturelle Kopplung von Recht und Politik in der transnationalen Konstellation gerade dadurch hergestellt werde, dass die Schiedsgerichte durch ihre Verweise auf völkerrechtliche und nationale zwingende Rechtsnormen „unterschiedliche Ebenen und Formen politischer Strukturbildung" reflektierten.

$\mathrm{Ob}$ das durch die Schiedsgerichte praktizierte einseitige und mehr oder minder willkürliche Bedienen aus einem Pool zwingender Normen unterschiedlicher Provenienz dem Anspruch auf demokratische Legitimation geltenden Rechts dabei tatsächlich genügt, mag gleichwohl bezweifelt werden. ${ }^{3}$ Dieser Umstand verweist auch auf das ungelöste Problem der Zirkularität des Rechts, welches auch Renners Ansatz begleitet: Die Entscheidungen der Schiedsgerichte werden anhand

3 Vgl. hierzu: Brunkhorst, Düstere Aussichten Die Zukunft der Demokratie in der Weltgesellschaft, Kritische Justiz 2010, S. 13 ff. einer Dogmatik des transnationalen zwingenden Rechts kritisiert, die auf Grundlage eben dieser Schiedspraxis entworfen wurde. Renners an Luhmann und Wiethölter orientierten Ausführungen zum Paradox der Rechtsbegründung können wir jedoch entnehmen, dass ihm eine solche Zirkularität eher als Auszeichnung denn als Makel einer Dogmatik gelten mag, schließlich lässt sich kaum bestreiten: „Kritik ohne Ersatzvorschlag zählt nicht. ${ }^{4}$

Insgesamt stellt das Werk Renners eine in jeder Hinsicht beeindruckende Leistung dar. Es überzeugt zunächst die auf einem reichen Schatz juristischer, philosophischer, soziologischer, politischer und ökonomischer Kenntnisse gegründete theoretische Durchdringung der hochkomplexen Strukturen eines entgrenzten, aber verflochtenen Rechts der Weltgesellschaft. Sodann der Bruch mit überkommenen Denkgewohnheiten und die Lösung der zentralen Herausforderungen durch einen Rückgriff auf den Topos des zwingenden Rechts sowie die Anknüpfung an das kollisionsrechtliche Denken Savignys. Schließlich auch die Sorgfalt, mit welcher Renner die Entscheidungen der Schiedsgerichte analysiert, die Theorie sich an der Praxis bewähren und an ihr wachsen lässt. Es beeindruckt aber nicht zuletzt auch die sehr eigene, kondensierte Darstellungsweise Renners. Präzise schreitet er den Grat zwischen maximaler intellektueller Dichte und notwendiger Allgemeinverständlichkeit ab: Niemals wird der Leser abgehängt, niemals wird er durch Abschweifungen gelangweilt. Kurzum: ein hervorragendes Werk.

Da sich nun im Werk schon kein Makel finden lässt, mag ein letzter Blick auf die Wirkung eines solchen Unternehmens gerichtet werden: Trägt die Entwicklung einer auf der Praxis aufbauenden Dogmatik angesichts des Legitimationsdefizits einer weitgehend autonomen Wirtschaftsverfassung nicht notwendig einen bitteren affirmativen Beigeschmack? Birgt diese Affirmation in Gestalt einer Systematisierung nicht dieselbe Gefahr, die Renner in Teubners Ansatz der Zivilverfassungen erblickt, nämlich dem sich tatsächlich vollziehenden Prozess einer schleichenden Entdemokratisierung des Rechts den juristischen Ritterschlag zu erteilen? Dass der Absicht des Autors nichts ferner liegen dürfte als eben dies, wird durch seine Ausführungen hinreichend deutlich. Es scheint vielmehr so zu sein,

4 Fischer-Lescano/Teubner, Prozedurale Rechtstheorie: Wiethölter, in: Buckel/Christensen/ Fischer-Lescano (Hrsg.), Neue Theorien des Rechts, 2. Aufl., 2009, S. 75 ff. (86). 
dass seinem Vorgehen eine unausgesprochene, aber dezidiert pragmatische Haltung zugrunde liegt, welche, statt sich mit Lamenti über die entfesselte Wirtschaftslogik der globalisierten Welt zu begnügen, eine optimale Anschlussfähigkeit der entwickelten Grundsätze an die realen Bedingungen der gegenwärtigen transnationalen Konstellation anstrebt. Fern jeglicher Schwärmerei von einer idealen „Weltrepublik“ (Höffe), gibt Renner dem heutigen Schiedsrichter das in konkrete, unmittelbar anwendbare Rechtsbegriffe und Vorrangregeln gegossene Instrumentarium an die Hand, um auch transnationale Fälle unter Berücksichtigung des Gemeinwohls zu lösen. Diese behutsame Vorgehensweise einer Zivilisierung in kleinen Schritten lässt Renner nicht nur in der geistigen Tradition Savignys stehen, ${ }^{5}$ sie zeugt auch von zwei Eigenschaften, die nur wenigen Rechtstheoretikern nachgesagt werden können: Realitätssinn und Bescheidenheit.

\section{Frederik von Harbou}

Oliver Eberl, Demokratie und Frieden Kants Friedensschrift in den Kontroversen der Gegenwart, Baden-Baden (Nomos-Verlag) 2008, 293 Seiten, $€ 39,00$

„Kein Staat soll sich in die Verfassung und Regierung eines andern Staats gewalttätig einmischen." Dieser Präliminarartikel aus Immanuel Kants Friedenschrift „Zum ewigen Frieden" ist im Zuge sog. humanitärer Interventionen und im Krieg gegen den Terror von der westlichen Staatenwelt missachtet worden. Oliver Eberls Abhandlung zu „Demokratie und Frieden - Kants Friedensschrift in den Kontroversen der Gegenwart" kritisiert die ideologische Offensive, die in Völkerrecht, internationalen Beziehungen und politischer Theorie seit den 1990ern daran arbei-

5 Vgl. Savigny (Fn. 2, Vorrede) zur Begründung des modernen IPR: „So finden wir also hier von der einen Seite die großartigsten Aussichten in die Zukunft, von der anderen Seite die Unmöglichkeit, die vorliegende Aufgabe schon jetzt zu einem vollständigen Abschluß zu führen [...]. Jeder, der sich in solcher Stellung befindet, kann aus dieser Betrachtung eben so viel Muth, als Bescheidenheit schöpfen. Er muß es sich zur Ehre rechnen, wenn es ihm gelingt, den fortgehenden geistigen Prozeß durch Zurückführung dieser Lehre auf eigentliche Grundsätze weiter fördern zu helfen, selbst wenn sein Versuch, bei fernerer Entwicklung, nur noch als einzelner, vorbereitender Schritt im Andenken bleiben sollte.". tet, aus dem pazifistischen Impuls der Kantischen Friedensschrift eine Ermächtigungsnorm zur kriegerischen Intervention zu gewinnen. Demgegenüber zeigt Eberl, dass solche „spezifisch liberalen Doktrinen der Intervention " (138) einer verzerrten Rezeption der Friedensschrift aufsitzen. Er legt eine eigene Rekonstruktion vor, in deren Mittelpunkt die Kantische Idee einer friedlichen Verrechtlichung der internationalen Staatenbeziehungen steht. Mit dieser präzise durchgearbeiteten Verbindung von Ideologiekritik und eigener, pazifistischer Lesart der Kantschen Friedensschrift hebt sich Eberl von einer Diskussionslandschaft ab, die zwischen dem utopischen Gehalt einer friedlichen Weltföderation und der Rechtfertigung hegemonialen Machtstrebens in Kantischen Kategorien hin und her gleitet. Eberls Hauptimpuls ist der Umstand, dass das Projekt einer Verrechtlichung der internationalen Staatenbeziehungen bei Kant kategorisch auf kriegerische Intervention verzichtet. Demgegenüber zeigt er, wie das liberale Völkerrecht, die internationalen Beziehungen sowie kosmopolitische Theorien der Weltbürgergesellschaft stets doch kriegerische Mittel in Kauf nehmen und deshalb an dem Kantschen Hauptanliegen vorbeisteuern. Eberl situiert Kant - um die Sprache des Völkerrechtlers Martti Koskenniemi zu bemühen (siehe ders., From Apology to Utopia. The structure of international legal argument, Cambridge 2006) - jenseits von Apologie und Utopie, jenseits von hegemonialer Legitimationsideologie und utopischem Idealzustand.

Zuerst wendet sich die Studie einer Kritik des zeitgenössischen Liberalismus zu. Der ideologiekritische Durchgang beginnt mit einer Kritik an der These vom demokratischen Frieden, wie sie Politikwissenschaften und Völkerrecht in das Kantsche Projekt hineinlesen: dass nämlich nur Demokratien untereinander in einem starken Sinne friedensfähig sind. In diesem diskursiven Feld des „neuen Liberalismus" stellt Eberl zwei fatale Fehlschlüsse fest: Zum einen sehen die neuzeitlichen Liberalen fälschlicherweise in den real existierenden Demokratien schon de facto das Gebot der Republikanisierung in der Friedensschrift realisiert. Doch bei Kant enthält das Republikanisierungsgebot insbesondere eine Abstimmung aller Bürger im Falle einer Kriegsentscheidung. Deshalb, so folgert Eberl, könne „von einer Unterwerfung der Militärmacht unter demokratischer Kontrolle" in den meisten Demokratien noch keine Rede sein (94). Zum anderen eröffnet diese vorschnelle Identifizierung von real existierenden Demokratien und Kantscher Repu- 
blik die Spielräume dafür, die Friedensneigung der Republik zu einer Ermächtigungsnorm für militärische Intervention umzudeuten. Der neue Liberalismus interpretiert Kants friedensstiftenden Völkerbund so, dass er ausschließlich aus Republiken besteht. Militärische Interventionen zur globalen Durchsetzung der liberalen Ordnung erhalten in dieser Perspektive eine eigentümliche Legitimität. Eberl entdeckt hier eine „Rechtsvergessenheit" (98), da Kant eindeutig für eine egalitäre Verrechtlichung der Staatenbeziehungen plädiert (2. Definitivartikel der Friedensschrift), die einem inklusiven Gleichbehandlungsgrundsatz aller Staaten (unabhängig von ihrer Herrschaftsform) und dem kategorischen Ausschluss militärischer Intervention folgt (120).

Nach dieser Kritik am neuen Liberalismus diskutiert die Abhandlung kosmopolitische Rezeptionsweisen der Friedensschrift, etwa von David Held, Ottfried Höffe oder Jürgen Habermas. Sie alle stellen den dritten Definitivartikel der Friedensschrift - die Gewährleistung eines Weltbürgerrechts - in den Mittelpunkt ihrer Argumentation. Der Staatenbund avanciert in dieser Perspektive zu einem weltweiten Bürgerbund, der sich auf die globale Menschenrechtsgeltung stützt. Doch auch hier arbeitet Eberl zwei problematische Fehlschlüsse heraus: Zum einen neigt der Kosmopolitismus dazu, die Menschenrechte dem Demokratieprinzip vorzulagern. Dadurch werde „die Idee des Schutzes der Menschenrechte durch die Demokratie, in der die Träger der Rechte selbst die Interpreten ihrer sind (...) aufgegeben" (155). Zum anderen erscheint die militärische Intervention hier als weltinnenpolitische Maßnahme, deren Umsetzung nicht mehr andere Staaten, sondern Institution jenseits der Nationalstaaten -z.B. die Vereinten Nationen - gewährleisten. Dadurch ergeben sich deutliche Widersprüche mit dem Konzept eines verrechtlichten Völkerbundes, der militärische Gewaltanwendung kategorisch ausschließt. Als ein Resultat des ideologiekritischen Durchgangs hält Eberl fest: „Der liberale Interventionismus erklärt sich nicht aus der Friedensschrift, sondern findet dort seinen Gegenspieler" (118). Um diesen Befund zu untermauern, legt Eberl eine eigene Rekonstruktion der Friedensschrift vor. Sie ist am radikaldemokratischen Kantianismus von Ingeborg Maus geschult und stellt die Rechtsdimension in den Mittelpunkt. Dazu gehört der Gleichbehandlungsgrundsatz und das „Existenzrecht aller existierenden Staaten“, die durch Verrechtlichung und konsequentes Interventionsverbot in einem Völkerbund assoziiert sind (185). Darü- ber hinaus zeigt Eberl, dass das Theorem einer Friedensneigung der Republiken nicht zu einem außenpolitischen Zivilisierungskreuzzug uminterpretiert werden kann; vielmehr speist sich die Friedensneigung der Republiken daraus, dass sie innenpolitisch auf einen demokratischen Entscheidungsmodus in Kriegsfragen zurückgreifen: Wenn das Volk selbst über Krieg und Frieden entscheidet, wird der Krieg unwahrscheinlicher. Eberl demonstriert, dass Kant bei dieser Annahme nicht vorrangig den Bürger als Bourgeois im Blick hat, der aus Angst vor materiellen Verlusten gegen den Krieg optiert. Eher ist es der „Zusammenhang von despotischer Gewalt und Kriegsneigung“ (189), also Machtkonzentration, Ausnahmezuständen und das Primat der Exekutiven, die den Kriegsbeschluss in einer Republik unwahrscheinlicher macht: „Die Entscheidungshoheit sichert nicht alleine den Besitz, sondern zuerst das Leben des homme, dann die Freiheit des citoyen und schließlich das Eigentum des Bourgeois“ (192). Schließlich interpretiert Eberl den dritten Definitivartikel Kants zum Weltbürgerrecht als Versuch, eine Verrechtlichungslücke des Völkerbundes zu schließen. Das Weltbürgerrecht soll die „Durchlässigkeit der Nationalstaaten für Individuen“ (244) garantieren. Es habe nicht - wie kosmopolitischen Ansätzen insinuieren - „supranational über die Einhaltung von Menschenrechten oder Demokratie zu wachen" (257).

Die Abhandlung argumentiert glasklar und zeigt den ideologischen Charakter der neueren Kant-Rezeption auf. Das ist wahrlich „nicht Nichts“ (Hegel). Denn die Zustimmungsfähigkeit von Kriegseinsätzen wurde mit den Argumenten des neuen Liberalismus, des Menschenrechtlichen und Universellen hergestellt. Dagegen weist Eberl schlüssig nach, dass die Aufklärungsphilosophie Kants gerade nicht eine Urform des modernen Bellizismus abgibt. Im Gegenteil: „Die unilaterale Selbstermächtigung dagegen kann nicht als dem Kantschen Projekt zugehörig begriffen werden" (211). Das streng Kantische in Eberls Argumentation ist die Stärke und die Schwäche der Abhandlung. Am Ende plädiert Eberl für den friedlichen Völkerbund souveräner Nationalstaaten. Aktuelle Befunde zur Internationalisierung von Produktionsbeziehungen und zur Internationalisierung der Staatsapparate (internationale politische Ökonomie), der sozialen Subsysteme (Systemtheorie), globaler Kommunikationsstrukturen (Neo-Institutionalismus) und zur Erosion nationalstaatlicher Souveränität, die eine solchen souveränitätsbasierten Ansatz unterlaufen, bleiben leider außen vor. Eberl ergreift 
konsequent für ein „tatsächlich liberales Programm" Partei (262). Der Gegenspieler seines kriegsfreien nationalstaatlichen Völkerbundes ist der interventionsschwangere Völkerbund des neuen Liberalismus. Man fragt sich, ob nicht auch ein Gegenentwurf zum liberalen Kosmopolitismus eine friedensschriftkompatible Option wäre - also eine radikaldemokratische Verrechtlichung des Bürgerbundes, die dem Prinzip der Nicht-Intervention folgt. Aber solche Überlegungen laufen vielleicht Gefahr, die Pointe der Eberlschen Argumentation zu verwässern: Der Nachweis, dass Kant in seiner Friedensschrift im Gegensatz zu seinen neueren Rezipienten gänzlich auf kriegerische Zivilisierungsbestrebungen verzichtet hatte.

Kolja Möller

Martti Koskenniemi, From Apology to Utopia. The Structure of International Legal Argument, Cambridge (Cambridge University Press) 1989, 2. Aufl. 2006, 683 S., 38,99 Euro; ders., The Gentle Civilizer of Nations: The Rise and Fall of International Law 1870-1960, Cambridge (Cambridge University Press) 2001, 569 S., 93 USD

I.

1989, im Jahr des großen Umbruchs, wird wohl eher Zufall als List der Vernunft - unter dem Titel „From Apology to Utopia. The Structure of International Argument" eine Dissertation publiziert, die nicht die Welt, aber gewiss ein Leben und ebenso gewiss eine Disziplin, das Völkerrecht, verändert. Das Leben betrifft den Autor, Martti Koskenniemi (1953), damals in der Mitte seiner Karriere als Diplomat und Repräsentant Finnlands in internationalen Organisationen und Konferenzen. Der Rechtsberater finnischer Delegationen - vor allem in der Generalversammlung, im Sicherheitsrat und in der Menschenrechtskommission der Vereinten Nationen wird, nicht unüblich im Rahmen einer Karriere als Diplomat und Politiker, bald zum amtierenden Direktor der Abteilung für internationales Recht im Außenministerium ernannt. Mit der Promotion schließt er jedoch nicht nur seine Juristenausbildung ab, sondern nimmt eine - wohl seit langem angestrebte, aber nicht geplante -, für sein Leben und seine Laufbahn entscheidende Weichenstellung vor: „From Apology to Utopia“ gibt deutlicher als den Praktiker des Völkerrechts und Recht-Fertiger nunmehr den kritischen Rechtswissenschaftler zu erkennen.

Martti Koskenniemi steigt in den folgenden Jahren zu einem der wobl einflussreichsten und dem gewiss am häufigsten zitierten und diskutierten Mitglieder seiner Zunft auf. Mit der Professur für internationales Recht an der Universität Helsinki verlässt er 1994, wie es zunächst scheint: endgültig, die Sphäre diskreter diplomatischer Rechtsberatung und vornehm exekutivischer Rechtsanwendung. Er wechselt über die Systemgrenze hin zu den zwangsläufig offenen Kontroversen wissenschaftlicher Beschäftigung mit dem Völkerrecht. 1997 übernimmt er zusätzlich als Direktor die Leitung des Erik-Castrén-Instituts für internationales Recht und Menschenrechte der Universität Helsinki. Es folgen, man ist versucht zu sagen: Schlag auf Schlag, ehrenvolle Berufungen auf Gastprofessuren an namhaften Universitäten - Brüssel; Paris II, X, XIII und I; The Hague Academy of International Law; Harvard/MA und Cambridge/ UK, um nur die wichtigsten zu nennen. Seit 1997 lehrt er immer wieder „in residence“ als Global Professor an der New York University School of Law. Ergänzt werden die Berufungen in akademische Beiräte und politische Beratungsgremien - also doch immer wieder Rückkehren in die Praxis. Es entbehrt nicht einer gewissen Ironie, dass der kritische Wissenschaftler, der eben noch das Völkerrecht in seine Bestandteile zerlegt hat, nun vier Jahre lang als Chairman der „Study Group: Fragmentation of International Law" und als Mitglied der International Law Commission der Vereinten Nationen fungiert. Seine Passion, trotz intensiver Lehr- und Vortragstätigkeit, ist Forschung, vor allem die Erforschung der Geschichte seiner Disziplin.

\section{II.}

„From Apology to Utopia“ ist in erstaunlich kurzer Zeit weltweit ein durchschlagender Erfolg beschieden. Vielleicht, weil Martti Koskenniemi eine neue intellektuelle Stimme und Perspektive in den wissenschaftlichen Diskurs über das Völker- bzw. internationale Recht einführt: dessen kritische Analyse als professionelle Praxis und akademische Disziplin des Argumentierens. Informiert durch seine eigenen, jahrelangen praktischen Erfahrungen, weiß er, wovon er spricht und schreibt, wenn er die Sprache und Argumente der Theorien und Doktrinen des Völkerrechts, wie sie "gelebt" werden, eingehend untersucht, ja, seziert. Diplomatisch in der 
Form, entschieden in der Sache, bricht er ${ }^{1}$ mit einer ehernen Tradition der Zunft, die von einem Lehrer des Völkerrechts, einem jungen zumal, will er über die nationalen Grenzen hinaus in seiner Disziplin Gehör finden, erwartet, sich mit einer neuen und einflussreichen Lösung eines klassischen Problems, wie etwa Souveränität, Gewaltverbot, Individualschutz im Völkerrecht etc., als einer, der Meinungsführerschaft anstrebt, vorzustellen. Wohl wissend, wie alle Mitglieder der völkerrechtlichen „Interpretationsgemeinschaft", dass sich diese vermeintlich "neue Lösung“ alsbald wieder entweder als eine alte Doktrin im neuen Gewande oder aber als unzulänglich, widersprüchlich, fehlerhaft etc. erweisen dürfte.

Martti Koskenniemi widersetzt sich den Regeln des eingerichteten und ausgeübten Dogmatikbetriebes. Statt ihnen zu gehorchen, dringt er mit seiner strukturellen Analyse des Vokabulars und der Grammatik völkerrechtlicher Lehren zu den widerstreitenden Motiven vor, von denen sich Lehrer (und Praktiker) des Völkerrechts bei ihrem disziplinären Sprachgebrauch immer wieder leiten lassen: nämlich von der doppelten Sehnsucht nach Rechtfertigung und Ratifizierung des Bestehenden einerseits, nach Verheißung einer Utopie - und sei sie auch noch so bescheiden - andererseits. Mit der Wendung hin zu den Strukturen, die in die Sprache des Völkerrechts und der Völkerrechtler eingelassen sind, ersetzt er die herkömmlichen Gegensätze zwischen Formalismus und Realismus, Positivismus und Naturalismus etc. durch eine Suche nach den Bedingungen der Möglichkeit völkerrechtlichen Argumentierens und damit einer „meaningful international legal practice" (S. 563).

Koskenniemis ernüchterndes Ergebnis seiner Analysen der Rechtfertigungsnarrative (insbesondere der Varianten des Liberalismus) und Leitbegriffe (etwa Souveränität, Rechtsquellen, ius cogens, Völkervertrags- und Völkergewohnheitsrecht): Die Grammatik der Sprache des Völkerrechts hat sich seit dem Ende des 19. Jahrhunderts kaum geändert; das Völkerrecht oszilliert zwischen rigorosem juridischem Formalismus und politisch-realistischer Offenheit (open-endedness), zwischen Normativität und Faktizität/Konkretheit, zwischen Apologie und Utopie. Statt nach einem „dritten Weg“, einem neuen Vokabular oder einer neuen Rechtstechnik zu suchen, behandelt Koskenniemi die Spannung zwi-

1 Wie der „Strukturalist“ David Kennedy in seiner Dissertation International Legal Structures, Baden-Baden 1987. schen Formalismus und Realismus als die übliche Erfahrung, die jeder Völkerrechtler im akademischen oder praktischen Kontext macht, weil es sich um eine generative Grammatik handelt. Offensichtlich traf „From Apology to Utopia“ damit einen zentralen Nerv in der Scientific Community des (Völker-)Rechts. Anders ist der Erfolg des Buches kaum zu erklären, das sich in kurzer Zeit zu einem Standardwerk der kritischen Analyse des Völkerrechts entwickelte. Das Erfolgsgeheimnis könnte sein, dass es nicht an Stimmen fehlte, die entweder den linguistischen Ansatz für zu wenig kritisch oder die zentrale Aussage „International law ist what international lawyers make of it" für zu radikal hielten.

\section{III.}

Es bleibt ein Rätsel, wie und wo Martti Koskenniemi trotz seiner akademischen Reise-, Vortrags- und Lehrtätigkeit die Zeit findet, die schier unüberschaubare Vielzahl von Aufsätzen, Buchbeiträgen, Entscheidungsanmerkungen und Rezensionen zu schreiben. Sie stellt nicht nur eine ungeheure Produktivität und Originalität unter Beweis, sondern dokumentiert zugleich, dass er nicht immer, aber immer wieder die in "From Apology to Utopia“ aufgeworfenen Fragen und Themen aufgreift und weiterentwickelt.

In den neueren Texten folgt er seinem „linguistic turn", es kommt jedoch zu zwei markanten, perspektivischen Verschiebungen: Zum einen richtet er den analytisch-kritischen Blick weniger auf die Strukturen als auf die Geschichte/Kontexte. Etwa fünfzehn Jahre nach "From Apology to Utopia“ legt Martti Koskenniemi seine zweite große Studie zum Völkerrecht vor: „The Gentle Civilizer of Nations. The Rise and Fall of International Law 1870-1960", die in kurzer Zeit, man ahnt es, wiederum einen Standard setzt. Nunmehr für die wissenschaftliche Befassung mit der Geschichte des Völkerrechts. Er erzählt in dieser wissenschaftlich anspruchsvollen, dennoch fesselnd geschriebenen Studie die Geschichte sowohl des Völkerrechts als Disziplin wie auch der Völkerrechtler als professioneller Klasse. Ähnlich wie in „From Apology to Utopia“ interessiert ihn die eigentümliche Verschwisterung von formaler Kohärenz und materialer Unbestimmtheit in den völkerrechtlichen Theorien, Lehren und Doktrinen und deren kontinuierliches Oszillieren zwischen den Polen von Formalismus und AntiFormalismus. Allerdings transformiert er in "The Gentle Civilizer of Nations" die Strukturanalyse in eine Serie von Narrativen. Den Aufstieg des Völkerrechts etwa seit 1870 begleitet er zu dessen Kompromittierung und 
Instrumentalisierung durch pragmatische, realpolitische Erwägungen, die an Weber'sche Materialisierungen der formalen Rationalität des Rechts erinnern und am Ende das Völkerrecht an eine Politologie der internationalen Beziehungen ausliefern.

Der Aufstieg beginnt mit der Gründung des Institut de Droit International und dessen Organ, der Revue de Droit International et Comparée, beide getragen von der emphatisch-humanistischen Idee, Ideologie und Utopie des Völkerrechts als des „rechtlichen Gewissens der zivilisierten Welt“. Die Geschichte des Institut und der Revue beschreibt Koskenniemi als eine Geschichte des Scheiterns, für die er die ambivalente Argumentation, den philosophischen Dilettantismus und die soziologische Ignoranz (von Rolin, Westlake, Asser, Bluntschli und anderen) haftbar macht. Bereits gegen Ende des 19. Jahrhunderts zeichnet sich mit dem ersten (Sünden-?)Fall das Ende dieser Utopie ab. Mit der Apologie des Empire und Imperialismus der Kolonialzeit verstricken sich die „Founding Fathers" auf der Ebene des völkerrechtlichen Begleitdiskurses in den "scramble for Africa“ und geben unfreiwillig das gefährliche „Supplement" (Derrida) jenes Humanismus und Universalismus der Gründerzeit zu erkennen - einen militanten Eurozentrismus, der wie ein Schatten, bisweilen als rassistischer Subtext, jenes „rechtliche Gewissen der zivilisierten Welt" begleitet. Die zivilisierende Mission Europas entpuppt sich in den beiden letzten Dekaden des 19. Jahrhunderts und in der Mandatspolitik nach dem Ersten Weltkrieg als blanke Ideologie, die nach der Logik von Exklusion/Inklusion Europas Rolle als Türhüter der von Macht und Wohlstand ausgeschlossenen kolonialen und später formal dekolonisierten Welt sanktioniert. ${ }^{2}$

Ideen und Ideologeme, Argumente und Theorien einflussreicher Lehrer und Schulen des Völkerrechts liest Martti Koskenniemi in den folgenden Kapiteln (S. 179 ff.) in ihre jeweiligen historischen und biographischen Kontexte ein. Er präpariert in dichten Erzählungen den radikalen Bruch heraus, der die Entfaltung eines neuen professionellen Selbstbewusstseins und Enthusiasmus zwischen 1869 und 1885 zunehmend von dessen formalem, wenn nicht effektivem Ende 1960 trennt. Unter dem Titel „International law as philosophy“ zeichnet Koskenniemi nachfol-

2 Ausführlich dazu die eindrucksvolle Studie von Anthony Anghie, Imperialism, Sovereignty, and the Making of International Law (2005); die Hauptthesen finden sich in: ders., Die Evolution des Völkerrechts, KJ 1/2009, 49. gend im Gegenlicht der französischen Schule („International law as sociology“) des Traditionalismus von Hersch Lauterpacht sowie der europäischen, von Carl Schmitt und Hans Morgenthau angeführten realistischen Wende, die Linien der völkerrechtlichen Lehren in Deutschland von 1871 bis 1933 (vertreten u.a. durch von Liszt, Jellinek, Kelsen, Kaufmann, Schücking, Strupp) nach. Er arbeitet heraus, dass die unterschiedlichen philosophischen Orientierungen zwischen Naturalismus und Positivismus, zwischen Formalismus und soziologischer Jurisprudenz oszillieren, ohne je zur Ruhe zu kommen oder gar in die Außenpolitik der krisengeschüttelten Weimarer Republik übersetzt zu werden. Am Ende löscht das faschistische Nazi-Regime die philosophischen Ansätze utopischer Visionen buchstäblich aus.

Die zweite Akzentverschiebung zeigt sich in Martti Koskenniemis Texten der letzten Jahre als vorsichtige Suche nach einer „Kultur des Formalismus", ${ }^{3}$ mit der er das normative Projekt von „From Apology to Utopia“ fortführt: ,to provide resources for the use of international law's professional vocabulary for critical or emancipatory causes". ${ }^{4}$ Die Existenz einer solchen Kultur stellt er im Schlussakkord von "The Gentle Civilizer of Nations" noch unter ein Fragezeichen, wohl um sich nicht vorwerfen zu lassen, den Widerspruch von Apologie und Utopie rekapituliere er in seiner eigenen Arbeit als Gegensatz von Kritik und Utopie. Ersichtlich geht es ihm auch in "The Gentle Civilizer of Nations" nicht darum, eine sichere Mittelposition $\mathrm{zu}$ finden zwischen einer soziologischen Beschreibung dessen, was (im Völkerrecht) Sache ist, und einer normativen Spekulation darüber, was völkerrechtlich gut wäre.

Nach getaner Kritik wendet sich Martti Koskenniemi am Ende mit eindrucksvollen Argumenten gegen die Reduktion von Recht, zumal von Völkerrecht, auf ein bloß technisches Instrumentarium und verteidigt die Auffassung, dass Recht mehr ist als (leere) Worte. Er lässt sich, wenn nicht von der Annahme, so doch der Vermutung leiten, dass die Berufung auf „Recht“ in internationalen Angelegenheiten - statt Politik, Moral oder Ökonomie etc. - einen Unterschied macht. Die schwierige Frage ist allerdings: welchen? Seine zwangsläufig tentative Antwort greift ein Leitmotiv von „From Apology to Utopia“ und "The Gentle Civilizer of Nations" auf: Die Vorstellung oder vielleicht regulative Idee einer Kultur des Formalismus verweist auf die

3 The Gentle Civilizer of Nations, S. $494 \mathrm{ff}$.

4 From Apology to Utopia, S. 589. 
Bedingungen der Möglichkeit von Recht und rechtlicher Argumentation. Sie steckt den Horizont für eine Praxis des Widerstandes gegen jede Form von Macht und gegen soziale Praktiken ab, die (auch für Kritik) offene wissenschaftliche Diskurse unter Gleichen unmöglich machen.

Martti Koskenniemi wird mit den Vorwürfen leben können, auch diese große Studie sei akritisch, weil sie der "scientific community“ wiederum keine transformative Perspektive auf ein vorzugswürdiges Set von internationalen Institutionen anbiete, oder aber sie sei unkritisch, weil sie mit dem bescheidenen Ziel eines moderaten Kosmopolitismus die Strukturkritik desavouiere. Für künftige Beiträge zu dem Genre „Leben und Werk von ..." und zur Geschichte des Völkerrechts hat er mit seinem vielfach ausgezeichneten „The Gentle Civilizer of Nations" inhaltliche und wegen der ebenso behutsamen wie kritischen Interpretationen wieder einmal hohe methodischtheoretische Maßstäbe gesetzt.

Günter Frankenberg 